\title{
Fast and accurate calculations for cumulative first-passage time distributions in Wiener diffusion models
}

Blurton, Steven Paul; Kesselmeier, M.; Gondan, Matthias

Published in:

Journal of Mathematical Psychology

DOI:

10.1016/j.jmp.2012.09.002

Publication date:

2012

Document version

Publisher's PDF, also known as Version of record

Citation for published version (APA):

Blurton, S. P., Kesselmeier, M., \& Gondan, M. (2012). Fast and accurate calculations for cumulative firstpassage time distributions in Wiener diffusion models. Journal of Mathematical Psychology, 56(6), 470-475. https://doi.org/10.1016/j.jmp.2012.09.002 


\title{
Fast and accurate calculations for cumulative first-passage time distributions in Wiener diffusion models
}

\author{
Steven P. Blurton ${ }^{\mathrm{a}}$, Miriam Kesselmeier ${ }^{\mathrm{b}}$, Matthias Gondan ${ }^{\mathrm{b}, *}$ \\ ${ }^{a}$ Department of Psychology, University of Regensburg, Germany \\ ${ }^{\mathrm{b}}$ Institute of Medical Biometry and Informatics, University of Heidelberg, Germany
}

\section{A R T I C L E I N F O}

\section{Article history:}

Received 20 April 2012

Received in revised form

20 August 2012

Available online 13 October 2012

\section{Keywords:}

Diffusion model

Wiener process

First passage times

Response times

\begin{abstract}
A B S T R A C T
We propose an improved method for calculating the cumulative first-passage time distribution in Wiener diffusion models with two absorbing barriers. This distribution function is frequently used to describe responses and error probabilities in choice reaction time tasks. The present work extends related work on the density of first-passage times [Navarro, D.J., Fuss, I.G. (2009). Fast and accurate calculations for first-passage times in Wiener diffusion models. Journal of Mathematical Psychology, 53, 222-230]. Two representations exist for the distribution, both including infinite series. We derive upper bounds for the approximation error resulting from finite truncation of the series, and we determine the number of iterations required to limit the error below a pre-specified tolerance. For a given set of parameters, the representation can then be chosen which requires the least computational effort.
\end{abstract}

(C) 2012 Elsevier Inc. All rights reserved.

\section{Introduction}

In the presence of two mutually exclusive competing risks, event times can often be described by a stochastic process drifting between two absorbing barriers. Typical examples include sequential sampling models of human decision making (e.g., Busemeyer \& Townsend, 1993; Diederich, 1997; Ratcliff, 1978; Ratcliff \& McKoon, 2008), or length of stay in hospital (with the two outcomes death and healthy discharge, e.g., Horrocks \& Thompson, 2004). The central assumption of these models is that a hidden underlying state randomly moves between two alternatives until eventually one of two criteria is reached (socalled absorbing barriers). The appeal in those models lies in the possibility to derive predictions not only for the probabilities for the two outcomes, but also for the time it takes until the barrier is hit. Often, this process is assumed to be continuous, resulting in the well-known diffusion models for which the time-homogeneous Brownian motion process (Wiener process, Fig. 1) is most popular.

The Wiener process $\mathbf{X}\left(t \mid v, \sigma^{2}\right)$ is described by two parameters $v$ and $\sigma^{2}>0$ representing the drift and variance (noise) of the process. The process is temporally and spatially homogeneous, that is,

\footnotetext{
Supported by the German Research Foundation (DFG, Grant GR 988/20-2 to Mark W. Greenlee and Matthias Gondan). Scripts written in R statistical language (R Core Team, 2012) and Matlab are provided as online supplementary material.

* Correspondence to: Institute of Medical Biometry and Informatics, Im Neuenheimer Feld 305, 69120 Heidelberg, Germany.

E-mail address: gondan@imbi.uni-heidelberg.de (M. Gondan).
}

drift and variance neither depend on the current state nor the time elapsed (e.g., Smith, 2000). In the two-alternative choice model, the process is assumed to start at $\mathbf{X}(0)=z$, and two absorbing barriers are assumed at zero and $a$, representing the two outcomes, $0<z<a$. Despite the relative simplicity of the process, it is hard to derive expressions for the density and distribution of the firstpassage times in the two-barrier situation. One must rather rely on infinite series (Wald, 1947). Of course, the evaluation of infinite series can only involve a finite number of terms. The series, however, are known to converge, and it is possible to estimate the error that results when calculation is stopped at a certain number of steps. The usual approach is to terminate the calculation when a desired level of accuracy is met, for example, if the absolute error is lower than some tolerance $\varepsilon>0$. This limit can be reached after evaluation of very few terms when convergence is good at the point where the function is evaluated. On the other side, at critical points, sufficient accuracy requires the calculation of several hundred terms or even more.

Two representations exist for the first-passage time density of a Wiener process between two absorbing barriers. These representations show different convergence behavior: While one representation converges quickly for small values of $t$, the other representation converges quickly for large values of $t$. Navarro and Fuss (2009) exploited these properties and provided a decision rule when to use the one or the other representation. The decision rule depends on the number of terms needed to achieve a predefined level of accuracy. Based on this idea, we propose a computationally efficient way to compute the cumulative firstpassage time distribution of a Wiener process between two absorbing barriers. 


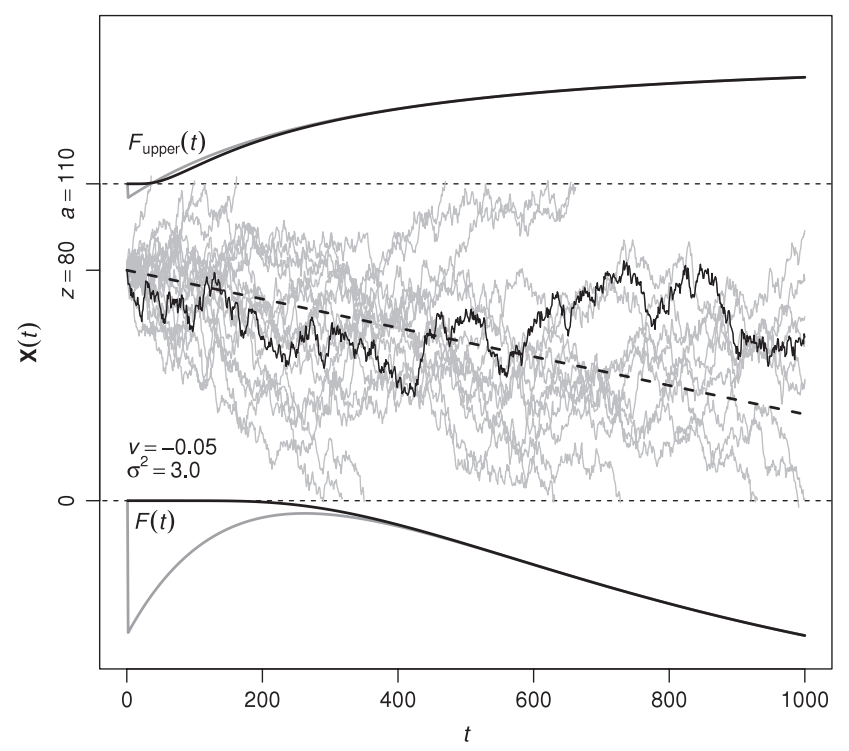

Fig. 1. Realizations of a Wiener process with variance $\sigma^{2}=3.0$ and drift $v=$ -0.05 (dashed line) starting at $z=80$ between two absorbing barriers at $a=110$ and zero. The black curves indicate the cumulative first-passage time distributions $F(t \mid v / \sigma, a / \sigma, w), w=z / a$, at the lower barrier and at the upper barrier [the latter is determined via $\left.F_{\text {upper }}(t \mid v / \sigma, a / \sigma, w)=F(t \mid-v / \sigma, a / \sigma, 1-w)\right]$. The gray lines show the approximation error resulting from early truncation at $K=2$.

\section{First-passage time density and distribution}

We consider a Wiener Process with drift $v$, starting at $\mathbf{X}(0)=z$. Without loss of generality, the variability can be fixed at $\sigma^{2}=1$, since it only scales the other parameters. A lower and an upper absorbing barrier is fixed at zero and $a$, with $0<z<a$. Setting, for convenience, $w=z / a$, the density $f(t)$ of first absorption at the lower barrier is described by the two series (e.g., Feller, 1968, p. 359 and p. 370),

$$
\begin{aligned}
f^{\ell}(t \mid v, a, w)= & \frac{\pi}{a^{2}} \exp \left(-v a w-\frac{v^{2} t}{2}\right) \\
& \times \sum_{k=1}^{\infty} k \sin (\pi k w) \exp \left[-\frac{1}{2}\left(\frac{k \pi}{a}\right)^{2} t\right], \\
f^{s}(t \mid v, a, w)= & \frac{a}{\sqrt{t^{3}}} \exp \left(-v a w-\frac{v^{2} t}{2}\right) \\
& \times \sum_{k=-\infty}^{\infty}(2 k+w) \varphi\left(\frac{2 k+w}{\sqrt{t} a),}\right.
\end{aligned}
$$

with $\varphi(x)$ denoting the standard normal density. Because absorption can occur at both the upper and the lower barrier, $f^{\ell}$ and $f^{s}$ are, in fact, subdensities and do not fully integrate to one. Whereas $f^{\ell}$ converges quickly for large $t, f^{s}$ converges quickly for small $t$ (Horrocks \& Thompson, 2004; Van Zandt, Colonius, \& Proctor, 2000). First absorption at the upper barrier is described by $f(t \mid$ $-v, a, 1-w)$; the lower density is given by $f(t \mid v / \sigma, a / \sigma, w)$.

Navarro and Fuss (2009) investigated the numerical properties of the two representations truncated at some $K$. In particular, they provide upper bounds for the error which results when the series $f^{\ell}, f^{s}$ are evaluated for $k=1, \ldots, K$ and $k=-K, \ldots, K$, respectively. They derived expressions for the required number of summands $K$ which limit the truncation error $\left|f(t)-f_{K}(t)\right|$ below a certain criterion $\varepsilon>0$ for each representation. For a prespecified set of parameters, the representation which is computationally least demanding can then be chosen.

In the present note we consider the cumulative first-passage time distribution, that is, the probability of absorption between time zero and some $t$, which is given by the integral of the density between zero and $t$. This distribution is again described by two alternative series with different convergence properties. Similar to Navarro and Fuss (2009) we derive upper bounds for the number of iterations $K$ required to limit the truncation error below a certain tolerance $\varepsilon>0$ for both representations. For a given set of parameters, the distribution is then determined using the representation which requires least computational effort. The decision is, thus, based on the number of iterations $K$, multiplied by the time it takes for each iteration.

\section{Large-time representation}

The large-time representation of the subdistribution of firstpassage times (e.g., Ratcliff and Tuerlinckx (2002), Eq. B1; Ratcliff (1978), Eq. A12) is obtained by integration of the large-time density $f^{\ell}(t)$ over $[0, t]$. Equivalently, the integral of $f^{\ell}(t)$ over $[t, \infty]$ is subtracted from the total probability $P$ of absorption at the lower barrier

$$
\begin{aligned}
F^{\ell}(t)= & P-\frac{2 \pi}{a^{2}} \exp \left(-v a w-\frac{v^{2} t}{2}\right) \\
& \times \sum_{k=1}^{\infty} \frac{k \sin (\pi k w)}{v^{2}+(k \pi / a)^{2}} \exp \left[-\frac{1}{2}\left(\frac{k \pi}{a}\right)^{2} t\right],
\end{aligned}
$$

with

$$
P= \begin{cases}\frac{1-\exp [-2 v a(1-w)]}{\exp (2 v a w)-\exp [-2 v a(1-w)]}, & \text { for } v \neq 0, \\ 1-w, & \text { for } v=0 .\end{cases}
$$

This workaround is necessary because term-wise integration of the infinite series $f^{\ell}(t)$ over $[0, t]$ requires uniform convergence of $f^{\ell}(t)$ within the range of integration, which can be demonstrated for positive $t$ only (Appendix A).

When determining $F^{\ell}(t)$, the series must be truncated at some $K \geq 1$. The number of summands $K$ should be chosen such that the truncation error $\left|F^{\ell}(t)-F_{K}^{\ell}(t)\right|$ is below some tolerance $\varepsilon>0$, that is,

$$
\begin{aligned}
& \mid \frac{2 \pi}{a^{2}} \exp \left(-v a w-\frac{v^{2} t}{2}\right) \\
& \quad \times \sum_{k=K+1}^{\infty} \frac{k \sin (\pi k w)}{v^{2}+(k \pi / a)^{2}} \exp \left[-\frac{1}{2}\left(\frac{k \pi}{a}\right)^{2} t\right] \mid \leq \varepsilon .
\end{aligned}
$$

To this end, two conditions for $K$ must be satisfied,

$K^{2} \geq \frac{1}{t}\left(\frac{a}{\pi}\right)^{2}$, and

$K^{2} \geq-\frac{2}{t}\left(\frac{a}{\pi}\right)^{2}\left\{\log \left[\frac{\varepsilon \pi t}{2}\left(v^{2}+\frac{\pi^{2}}{a^{2}}\right)\right]+v a w+\frac{v^{2} t}{2}\right\}$.

A detailed derivation is found in Appendix A. Briefly, the expression in (2) is simplified by omitting the sine and limiting $k$ at 1 in the denominator of the fraction behind the $\sum$. The exponential series is then replaced by an integral representing its upper bound and solved for $K$. As expected, the number of required terms increases monotonically with $\varepsilon$ and decreases with $t$-hence the name of (1), "large-time representation". For small $t, K$ tends to infinity (Fig. 2).

\section{Small-time representation}

The second representation of the cumulative first-passage time distribution is obtained by integration of the small-time density (e. g., Horrocks \& Thompson, 2004): 


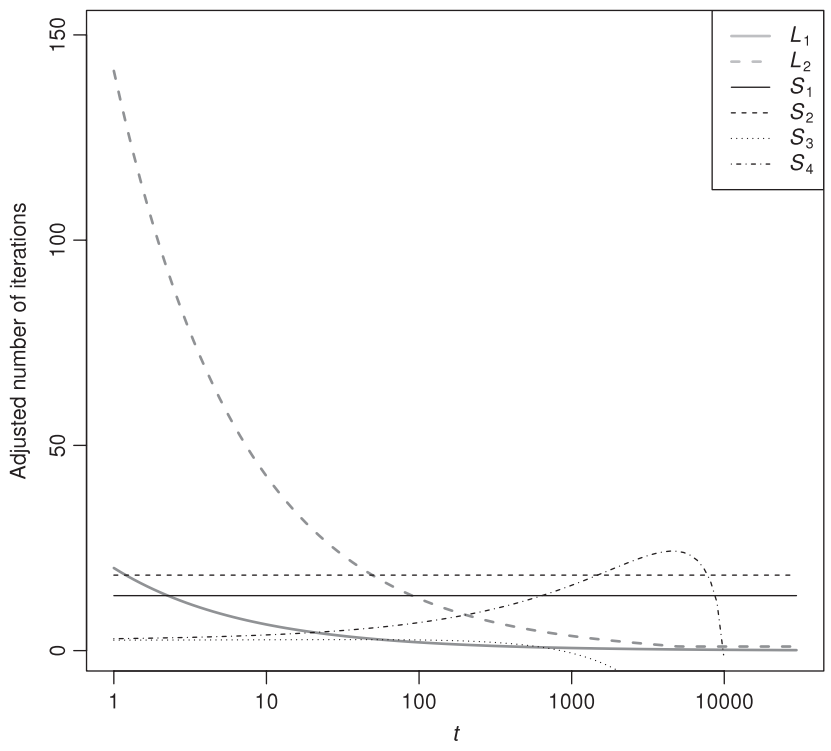

Fig. 2. Convergence of the two representations of the cumulative first-passage time distribution for $v=-0.06, a=63.2, w=0.5$, and $\varepsilon=1.5 \times 10^{-8}$ which is the square root of floating point precision with doubles. $L_{1}, L_{2}$ : Criteria for the largetime representation (3), with the subscripts denoting the square root of the criteria given in Appendix A. The required number of iterations is given by the ceiling of the maximum of $L_{1}$ and $L_{2} . S_{1}, S_{2}, S_{3}, S_{4}$ : Criteria for the small-time representation (5). The subscripts denote the respective expressions of Appendix B. The adjusted computational effort is again given by the ceiling of the maximum of $S_{1}, S_{2}, S_{3}$ and $S_{4}$, multiplied by 10 to account for the increased computing demands of the smalltime representation.

$$
\begin{aligned}
F^{s}(t)= & P-\operatorname{sgn} v \cdot \sum_{k=-\infty}^{\infty}[\exp (-2 v a k-2 v a w) \\
& \times \Phi\left(\operatorname{sgn} v \frac{2 a k+a w-v t}{\sqrt{t}}\right) \\
& \left.-\exp (2 v a k) \Phi\left(\operatorname{sgn} v \frac{-2 a k-a w-v t}{\sqrt{t}}\right)\right],
\end{aligned}
$$

with $P$ defined as in (1) and $\Phi(x)$ denoting the cumulative standard normal distribution. As before, the series in (4) describes the survivor function, that is, the probability for absorption between $t$ and infinity, such that the result is again subtracted from the probability $P$ of absorption at the lower barrier. Although this representation is undefined for $t=0, \lim _{t \rightarrow 0} F^{s}(t)$ can be shown to be zero, and the series shows good convergence for small $t>0$. Despite the name, convergence is acceptable for large $t$; the series is computationally expensive, however, for drift rates near zero.

We first consider negative drift $v<0$ (denoted by an additional superscript), that is, we are interested in a process with drift towards the lower barrier. Truncation of $F^{s-}(t)$ at some $K \geq 1$ yields a truncation error $\left|F^{s-}(t)-F_{K}^{s-}(t)\right|$ which should again be below $\varepsilon>0$,

$$
\begin{aligned}
& \mid \sum_{|k|=K+1}^{\infty}\left[\exp (2 v a k) \Phi\left(\frac{2 a k+a w+v t}{\sqrt{t}}\right)\right. \\
& \left.\quad-\exp (-2 v a k-2 v a w) \Phi\left(\frac{-2 a k-a w+v t}{\sqrt{t}}\right)\right] \mid \leq \varepsilon .
\end{aligned}
$$

As shown in Appendix B, three conditions must be satisfied for $K$,

$$
\begin{aligned}
& K \geq w-1+\frac{1}{2 v a} \log \left\{\frac{\varepsilon}{2}[1-\exp (2 v a)]\right\}, \\
& K \geq \frac{0.535 \sqrt{2 t}+v t+a w}{2 a}, \text { and }
\end{aligned}
$$

$K \geq \frac{w}{2}-\frac{\sqrt{t}}{2 a} \Phi^{-1}\left[\frac{\varepsilon a}{0.3 \sqrt{2 \pi t}} \exp \left(\frac{v^{2} t}{2}+v a w\right)\right]$.

As illustrated in Fig. 2, the first requirement dominates the criteria over a large range of $t$. Expression (4) is computationally more complex than the large-time representation (2). For a fixed $K$, repeated evaluation with different parameters showed $F^{s}(t)$ to be about ten times slower than $F^{\ell}(t)$.

For positive drift,

$$
\begin{aligned}
F^{s+}(t)= & P-\sum_{k=-\infty}^{\infty}\left[\exp (-2 v a w-2 v a k) \Phi\left(\frac{2 a k+a w-v t}{\sqrt{t}}\right)\right. \\
& \left.-\exp (2 v a k) \Phi\left(\frac{-2 a k-a w-v t}{\sqrt{t}}\right)\right] .
\end{aligned}
$$

As shown in Appendix B, the number of required summands can be determined using the criteria (5), with $v^{\prime}=-v$ instead of $v$ and a modified tolerance criterion $\varepsilon^{\prime}=\varepsilon \exp (-2 v a w)$.

In the zero drift case, the series simplifies to

$$
F^{s 0}(t)=2 \sum_{k=0}^{\infty}\left[\Phi\left(\frac{-2 a k-a w}{\sqrt{t}}\right)-\Phi\left(\frac{-2 a k-2 a+a w}{\sqrt{t}}\right)\right],
$$

and evaluation of $K \geq \frac{w}{2}-\frac{\sqrt{t}}{2 a} \Phi^{-1}\left(\frac{\varepsilon}{2-2 w}\right)$ terms guarantees a finite truncation error below $\varepsilon$.

\section{Discussion}

The present paper provides finite approximations of the cumulative first-passage times in the two-barrier diffusion model that controls the approximation error below a pre-specified tolerance. By comparing the required number of iterations in the two representations (2) and (4), and adjusting for the time necessary to evaluate a single summand of the series, the representation which requires least computational effort can be chosen. The present approach is to be preferred over ad hoc methods in which evaluation of the series is stopped when a single term is below the tolerance: When truncation is based on the absolute value of a single summand, the truncation error might be larger than expected because an infinite number of summands is dropped. To overcome this limitation, current implementations of the method sometimes evaluate a much larger number of summands than necessary. Here we propose to control the truncation error of the entire set of truncated summands. Precision is, therefore, controlled uniformly for all parameter combinations, which yields a smooth surface for numerical likelihood maximization (e.g., Horrocks \& Thompson, 2004).

In some applications, other parameter estimation procedures might be more suitable. For example, for the well known diffusion model (Ratcliff, 1978), an algorithm for calculation of the distribution function has been proposed by Voss and colleagues (Voss, Rothermund, \& Voss, 2004; Voss \& Voss, 2007, Eq. A9). The approach of Voss and colleagues is similar to ours, but they derive an expression for the required number of steps using the large-time representation (1) only. In the general case, this threshold is far too conservative, especially for small error bounds. Alternatively, discrete approximations (e.g., random walks) to continuous diffusion processes offer more complex, yet more flexible implementations of diffusion processes (Diederich \& Busemeyer, 2003).

Applications of the proposed method arise in fitting Ratcliff's (1978) diffusion model to observed response times, for example, from two-alternative choice tasks. Several methods have been proposed for this purpose, none of which can be said to be uniformly superior to the other methods (Ratcliff \& Tuerlinckx, 2002, pp. 443f). The so-called chi-square fitting method and 
the weighted least squares fitting method make heavy use of the cumulative first-passage time distribution $F(t)$. In contrast, likelihood maximization primarily uses the density $f(t)$ of the absorption times. In the latter approach, the distribution $F(t)$ is still needed in the presence of censored observations. Censoring occurs, for example, when the observer is unable to decide between two alternatives within a reasonable amount of time, or when responses are registered during a short time window in fixed stimulation protocols (e. g., in fMRI experiments). Then, absorption might be known to have occurred at the upper barrier, but it is only known to have occurred later than some $t$ ("misses"). A diffusion model with a deadline parameter could account for this, making use of the distribution $F(t)$, because the likelihood contribution then corresponds to the upper subsurvivor function at $t$. If absorption is only known to have occurred later than some $t$, and the outcome is unknown because no response has been given, the likelihood contribution corresponds to the sum of the upper and the lower subsurvivor function at $t$. The present method, thus, complements Navarro and Fuss' (2009) work on the density representation and will allow for the efficient parameter adjustment of diffusion models of competing risks even in the presence of censored observations.

\section{Appendix A. Integral and convergence of the large-time repre- sentation}

By collapsing the two exponentials, the density $f^{\ell}(t)$ is restated as a series of exponentials of $t$,

$$
\begin{aligned}
f^{\ell}(t)= & \frac{\pi}{a^{2}} \exp (-v a w) \\
& \times \sum_{k=1}^{\infty} k \sin (\pi k w) \exp \left\{-\frac{1}{2}\left[v^{2}+\left(\frac{k \pi}{a}\right)^{2}\right] t\right\} .
\end{aligned}
$$

Summand-wise integration of $f^{\ell}(t)$ over the interval $[\tau, \infty], \tau>0$ requires uniform convergence of $f^{\ell}(t)$ within that interval. This can be shown, for example, by the so-called majorant criterion (Weierstrass $M$-test). To this end, we define an upper bound $M_{\tau}$ for $f^{\ell}(t)$ with a small $\tau>0$, and drop the sine. Because $\sin x$ cannot exceed 1 and $\exp (-c t), c>0$, monotonically decreases in $t$,

$$
\begin{aligned}
& \left|f^{\ell}(t)\right| \leq M_{\tau}=\frac{\pi}{a^{2}} \exp (-v a w) \\
& \quad \times \sum_{k=1}^{\infty} k \exp \left\{-\frac{1}{2}\left[v^{2}+\left(\frac{k \pi}{a}\right)^{2}\right] \tau\right\}, \quad \text { for } t \geq \tau .
\end{aligned}
$$

The series $f^{\ell}(t)$ then converges if $M_{\tau}$ converges. Convergence of $M_{\tau}$ can be shown by the integral test because $M_{\tau}$ is positivevalued and strictly monotonically decreasing in $k$. As the integral $\int_{1}^{\infty} k \exp \left[-\frac{\tau}{2}\left(\frac{\pi}{a}\right)^{2} k^{2}\right] \mathrm{d} k$ exists and is finite, $M_{\tau}$ converges, such that $f^{\ell}(t)$ is uniformly convergent within $[\tau, \infty]$. Because all summands are exponentials of $t$, the antiderivative of $f^{\ell}(t), t \geq 0$, is easily found:

$$
\begin{aligned}
\int_{\tau}^{t} f^{\ell}(s) \mathrm{d} s= & -\frac{2 \pi}{a^{2}} \exp (-v a w) \\
& \times\left.\sum_{k=1}^{\infty} \frac{k \sin (\pi k w)}{v^{2}+\left(\frac{k \pi}{a}\right)^{2}} \exp \left\{-\frac{1}{2}\left[v^{2}+\left(\frac{k \pi}{a}\right)^{2}\right] t\right\}\right|_{\tau} ^{t} .
\end{aligned}
$$

The distribution function $F^{\ell}(t)$ is then obtained by subtracting the integral of $f^{\ell}(t)$ for $[\tau, \infty]$ from the total proportion $P$ of absorptions at the upper barrier,

$$
\begin{aligned}
F^{\ell}(t)= & P-\frac{2 \pi}{a^{2}} \exp \left(-v a w-\frac{v^{2} t}{2}\right) \\
& \times \sum_{k=1}^{\infty} \frac{k \sin (\pi k w)}{v^{2}+\left(\frac{k \pi}{a}\right)^{2}} \exp \left[-\frac{1}{2}\left(\frac{k \pi}{a}\right)^{2} t\right] .
\end{aligned}
$$

What happens if the series in (A.1) is truncated after evaluations of $K$ terms? In order to guarantee that the approximation error is below a certain tolerance $\varepsilon>0$, the absolute difference between the full series $F^{\ell}(t)$ and the truncated series $F_{K}^{\ell}(t)$ must be kept below the tolerance,

$$
\begin{aligned}
\left|F^{\ell}(t)-F_{K}^{\ell}(t)\right|= & \mid \frac{2 \pi}{a^{2}} \exp \left(-v a w-\frac{v^{2} t}{2}\right) \\
& \times \sum_{k=K+1}^{\infty} \frac{k \sin (\pi k w)}{v^{2}+\left(\frac{k \pi}{a}\right)^{2}} \exp \left[-\frac{1}{2}\left(\frac{k \pi}{a}\right)^{2} t\right] \mid \\
\leq & \varepsilon .
\end{aligned}
$$

To determine the conditions for $K$ some estimations have to be carried out. A first upper bound for $\left|F^{\ell}(t)-F_{K}^{\ell}(t)\right|$ is obtained by noting that $0 \leq|\sin x| \leq 1$ and by setting $k=1$ in the denominator as a lower bound for the fraction before the exponential. Thus, $\left|F^{\ell}(t)-F_{K}^{\ell}(t)\right| \leq \varepsilon$ if

$$
\begin{aligned}
& \frac{2 \pi}{a^{2}} \exp \left(-v a w-\frac{v^{2} t}{2}\right) \\
& \quad \times \sum_{k=K+1}^{\infty} \frac{k}{v^{2}+\left(\frac{\mathbf{1} \cdot \pi}{a}\right)^{2}} \exp \left[-\frac{1}{2}\left(\frac{k \pi}{a}\right)^{2} t\right] \leq \varepsilon .
\end{aligned}
$$

The factor before the sum is positive. Truncation of the series should, thus, be limited to those $K$ for which the elements decrease in $k$. The first derivative of the function $h(k)=k \exp \left[-\frac{1}{2}\left(\frac{k \pi}{a}\right)^{2} t\right]$ must, therefore, be negative, which is guaranteed if $K^{2}$ is greater than

$L_{1}=\frac{1}{t}\left(\frac{a}{\pi}\right)^{2}$.

Since the elements decrease, an upper bound for the error series $\sum_{k=K+1}^{\infty} h(k)$ is given by the integral of $h(k)$ within $K$ and infinity, so that

$$
\begin{aligned}
\left|F^{\ell}(t)-F_{K}^{\ell}(t)\right| \leq & \frac{2 \pi}{a^{2}} \exp \left(-v a w-\frac{v^{2} t}{2}\right) \\
& \times \frac{1}{v^{2}+(\pi / a)^{2}} \int_{K}^{\infty} h(k) \mathrm{d} k,
\end{aligned}
$$

which is below $\varepsilon$ if $K^{2} \geq L_{2}$ with

$$
\begin{aligned}
L_{2}= & -\frac{2}{t}\left(\frac{a}{\pi}\right)^{2}\left\{\log \left[\frac{\varepsilon \pi t}{2}\left(v^{2}+\frac{\pi^{2}}{a^{2}}\right)\right]\right. \\
& \left.+v a w+\frac{v^{2} t}{2}\right\} .
\end{aligned}
$$

For large $t$, the condition silently holds. In the other cases, $K$ is set to the ceiling of $\max \left(\sqrt{L_{1}}, \sqrt{L_{2}}\right)$.

In the zero drift case $v=0$, Expression (A.1) simplifies to

$$
F^{\ell 0}(t)=P-\frac{2}{\pi} \sum_{k=1}^{\infty} \frac{\sin (\pi k w)}{k} \exp \left[-\frac{1}{2}\left(\frac{k \pi}{a}\right)^{2} t\right] .
$$

The truncation error is controlled if the series is evaluated until $K^{2}$ is above $L_{1}^{0}=L_{1}$ and

$L_{2}^{0}=-\frac{2}{t}\left(\frac{a}{\pi}\right)^{2} \log \left(\frac{\varepsilon \pi^{3} t}{2 a^{2}}\right)$. 


\section{Appendix B. Convergence of the small-time representation}

We first consider negative drift $(v<0)$, denoted by an additional superscript. The truncation error of the small-time version of the subdistribution is most easily controlled by decomposing $F^{s-}(t)$ (which is known to be finite) into three distinct series:

$$
\begin{aligned}
F^{s-}(t)= & P-\sum_{k=0}^{\infty}\left[\exp (2 v a k) \Phi\left(\frac{2 a k+a w+v t}{\sqrt{t}}\right)\right. \\
& \left.-\exp (-2 v a k-2 v a w) \Phi\left(\frac{-2 a k-a w+v t}{\sqrt{t}}\right)\right] \\
& -\sum_{k=1}^{\infty} \exp (-2 v a k) \Phi\left(\frac{-2 a k+a w+v t}{\sqrt{t}}\right) \\
& +\sum_{k=1}^{\infty} \exp (2 v a k-2 v a w) \Phi\left(\frac{2 a k-a w+v t}{\sqrt{t}}\right) .
\end{aligned}
$$

All series are positive, the truncation error of the sum is, thus, guaranteed to be below the error tolerance $\varepsilon$ if the approximation error of each summand is controlled at $\varepsilon / 2$.

Denoting the inverse Gaussian distribution by $W(t \mid c, \mu)=$ $\Phi\left(\frac{\mu t-c}{\sqrt{t}}\right)+\exp (2 c \mu) \Phi\left(\frac{-\mu t-c}{\sqrt{t}}\right)$, the first series in Expression (B.1) can be rewritten as $\sum_{k=0}^{\infty} \exp (2 v a k)[1-W(t \mid 2 a k+$ $a w,-v)]$. Again, we truncate after $K$ summands have been evaluated. Because $W(t)$ is bounded between 0 and 1 , and $v$ is negative, $\exp (2 v a k)$ is recognized as a decreasing geometric series:

$$
\begin{gathered}
\sum_{k=K+1}^{\infty} \exp (2 v a k)[1-W(t \mid 2 a k+a w,-v)] \\
\leq \sum_{k=K+1}^{\infty} \exp (2 v a k)=\frac{\exp [2 v a(K+1)]}{1-\exp (2 v a)}
\end{gathered}
$$

The result is below the tolerance $\varepsilon / 2$ for $K$ greater than

$S_{1}=-1+\frac{1}{2 v a} \log \left\{\frac{\varepsilon}{2}[1-\exp (2 v a)]\right\}$,

independent of $t$. Similarly, the last series in (B.1) has converged for $K$ above

$S_{2}=w+S_{1}$,

which, of course, includes $S_{1}$.

In the second series in (B.1), large exponentials are multiplied with tiny $\Phi(-x)$, such that the product is finite. An upper bound for $\Phi(-x)$ is given by Ermolova and Haggman (2004),

$$
\begin{aligned}
\Phi(-x) & =\frac{1}{2} \operatorname{erfc}\left(\frac{x}{\sqrt{2}}\right) \leq 0.3 \exp \left(-1.01 \frac{x^{2}}{2}\right) \\
& \leq 0.3 \exp \left(-\frac{x^{2}}{2}\right) .
\end{aligned}
$$

The Ermolowa-Haggman bound requires the argument of $\operatorname{erfc}(\cdot)$ to be greater than 0.535 , which is satisfied if $K$ is greater than

$S_{3}=\frac{0.535 \sqrt{2 t}+v t+a w}{2 a}$.

Application of the bound to the second series in (B.1) yields exponentials decreasing in $k$, for which an upper bound is given by their integral. This integral is then recognized as a normal distribution:

$$
\sum_{k=K+1}^{\infty} \exp (-2 v a k) \Phi\left(\frac{-2 a k+a w+v t}{\sqrt{t}}\right)
$$

$$
\begin{aligned}
& \leq 0.3 \exp \left(-v a w-\frac{v^{2} t}{2}\right) \sum_{k=K+1}^{\infty} \exp \left[-\frac{(2 a k-a w)^{2}}{2 t}\right] \\
& \leq 0.3 \exp \left(-v a w-\frac{v^{2} t}{2}\right) \int_{K}^{\infty} \exp \left[-\frac{(2 a k-a w)^{2}}{2 t}\right] \mathrm{d} k \\
& =\frac{0.3}{2 a} \sqrt{2 \pi t} \exp \left(-v a w-\frac{v^{2} t}{2}\right) \Phi\left(\frac{a w-2 a K}{\sqrt{t}}\right) .
\end{aligned}
$$

The result is below $\varepsilon / 2$ if

$\Phi\left(\frac{a w-2 a K}{\sqrt{t}}\right) \leq \frac{\varepsilon a}{0.3 \sqrt{2 \pi t}} \exp \left(\frac{v^{2} t}{2}+v a w\right)$.

If the right hand side is larger than one, the condition silently holds. In the other cases, standard approximations for the quantile function of the normal distribution can be used to solve for $K$ which must be greater than

$S_{4}=\frac{w}{2}-\frac{\sqrt{t}}{2 a} \Phi^{-1}\left[\frac{\varepsilon a}{0.3 \sqrt{2 \pi t}} \exp \left(\frac{v^{2} t}{2}+v a w\right)\right]$.

For positive drift $v>0$,

$$
\begin{aligned}
F^{s+}(t)= & P-\sum_{k=-\infty}^{\infty}[\exp (-2 v a k-2 v a w) \\
& \times \Phi\left(\frac{2 a k+a w-v t}{\sqrt{t}}\right) \\
& \left.-\exp (2 v a k) \Phi\left(\frac{-2 a k-a w-v t}{\sqrt{t}}\right)\right],
\end{aligned}
$$

with truncation error

$$
\begin{aligned}
\left|F^{s+}(t)-F_{K}^{s+}(t)\right|= & \sum_{k=K+1}^{\infty}[\exp (-2 v a k-2 v a w) \\
& \times \Phi\left(\frac{2 a k+a w-v t}{\sqrt{t}}\right) \\
& \left.-\exp (2 v a k) \Phi\left(\frac{-2 a k-a w-v t}{\sqrt{t}}\right)\right] .
\end{aligned}
$$

The truncation error for positive drift, thus, corresponds to $\exp (2 v a w)$ times the error for negative drift, $\exp (2 v a w) \mid F^{s+}(t \mid v$, $a, w)-F_{K}^{s+}(t \mid v, a, w)|=| F^{s-}(t \mid-v, a, w)-F_{K}^{s-}(t \mid-v, a, w) \mid$. The required number of iterations for $v>0$ can, therefore, be determined using the expressions for $v^{\prime}=-v$ with a stricter criterion $\varepsilon^{\prime}=\varepsilon \exp (-2 v a w)$.

In the special case of zero drift, the series reduces to

$$
\begin{aligned}
F^{s 0}(t)= & 2 \sum_{k=0}^{\infty}\left\{\Phi\left[\frac{-2 a k-a+a(1-w)}{\sqrt{t}}\right]\right. \\
& \left.-\Phi\left[\frac{-2 a k-a-a(1-w)}{\sqrt{t}}\right]\right\} .
\end{aligned}
$$

The expression can be illustrated as series of bands of width $2(1-w)$ along the negative tail of a normal distribution (e.g., Fig 2.2 in Horrocks, 1999) with mean zero and variance $t / a^{2}$,

$F^{s 0}(t)=2 \sum_{k=0}^{\infty} \int_{-2 k-1-(1-w)}^{-2 k-1+(1-w)} \varphi\left(x \mid 0, \frac{t}{a^{2}}\right) \mathrm{d} x$,

such that the truncation error $\left|F^{s 0}(t)-F_{K}^{s 0}(t)\right|$ is below $2(1-w) \times$ $\Phi\left(-2 K+w \mid 0, t / a^{2}\right)$. The latter expression fulfills the tolerance criterion $\varepsilon$ if $K \geq \frac{w}{2}-\frac{\sqrt{t}}{2 a} \cdot \Phi^{-1}\left(\frac{\varepsilon}{2-2 w}\right)$.

\section{Appendix C. Supplementary data}

Supplementary material related to this article can be found online at http://dx.doi.org/10.1016/j.jmp.2012.09.002. 


\section{References}

Busemeyer, J. R., \& Townsend, J. T. (1993). Decision field theory: A dynamiccognitive approach to decision making in an uncertain environment. Psychological Review, 100, 432-459.

Diederich, A. (1997). Dynamic stochastic models for decision making under time constraints. Journal of Mathematical Psychology, 41, 260-274.

Diederich, A., \& Busemeyer, J. R. (2003). Simple matrix methods for analyzing diffusion models of choice probability, choice response times, and simple response time. Journal of Mathematical Psychology, 47, 304-322.

Ermolova, N., \& Haggman, S.G. (2004), Simplified bounds for the complementary error function; application to the performance evaluation of signal processing systems, Proceedings of the 12th European signal processing conference, 1087-1090.

Feller, W. (1968). An introduction to probability theory and its applications: Vol. 1 (3rd ed.). New York: Wiley.

Horrocks, J. (1999) Double barrier models for length of stay in hospital, Ph.D. thesis Waterloo, Canada.

Horrocks, J., \& Thompson, M. E. (2004). Modeling event times with multiple outcomes using the Wiener process with drift. Lifetime Data Analysis, 10, 29-49.
Navarro, D. J., \& Fuss, I. G. (2009). Fast and accurate calculations for first-passage times in Wiener diffusion models. Journal of Mathematical Psychology, 53, 222-230.

R Core Team, (2012). R: A language and environment for statistical computing. In $R$ Foundation for Statistical Computing. Austria: Vienna.

Ratcliff, R. (1978). A theory of memory retrieval. Psychological Review, 85, 59-108.

Ratcliff, R., \& McKoon, G. (2008). The diffusion decision model: theory and data for two-choice decision tasks. Neuronal Computation, 20, 873-922.

Ratcliff, R., \& Tuerlinckx, F. (2002). Estimating parameters of the diffusion model: Approaches to dealing with contaminant reaction times and parameter variability. Psychonomic Bulletin Er Review, 9, 438-481.

Smith, P. L. (2000). Stochastic dynamic models of response time and accuracy: A foundational primer. Journal of Mathematical Psychology, 44, 408-463.

Van Zandt, T., Colonius, H., \& Proctor, R. W. (2000). A comparison of two response time models applied to perceptual matching. Psychonomic Bulletin \&' Review, 7, 208-256.

Voss, A., Rothermund, K., \& Voss, J. (2004). Interpreting the parameters of the diffusion model: An empirical validation. Memory \& Cognition, 32, 1206-1220.

Voss, A., \& Voss, J. (2007). A fast numerical algorithm for the estimation of diffusion model parameters. Journal of Mathematical Psychology, 52, 19.

Wald, A. (1947). Sequential Analysis. New York: Wiley. 